\title{
Holism and Reductionism: How to Get the Balance Right
}

\author{
Alfred Abächerli \\ Lausanne, Switzerland \\ Email: fredaba@bluewin.ch \\ Received 25 April 2016; accepted 10 May 2016; published 13 May 2016 \\ Copyright (C) 2016 by author and OALib. \\ This work is licensed under the Creative Commons Attribution International License (CC BY). \\ http://creativecommons.org/licenses/by/4.0/

(c) (i) Open Access

\section{Abstract}

This is an extensive study of the conditions that allow or impede reductionism. After an overview of the different anti-reductionist conditions in approaches like holism, downward causation, emergence, supervenience, and several forms of uncertainty and sensitive dependence, a more detailed analysis of downward causation and holism is presented. For the latter, strong and numerous arguments could be identified why the whole is more than the sum of its parts. However, the examination of the case of official big bang theory, specifically a finite universe based on ultimate particles, allows the formulation of a potential reductionist theory in principle. The key notions for the further argumentation are different forms of infinity: the infinitely small, the infinitely big and the infinity of points in a continuous space-time, even if this is finite in its extension. Several cases are presented in which systems are at the same time holistic and reductionist if conditions of infinity are applied. This is identified as a natural ontological property of such systems as soon as infinity intervenes. The final conclusion, however, is that the certainties of reductionism are methodologically only useful on very small objects. In the macroscopic world the only way to influence things is by controlling or directing the processes by downward causation, which is based on the elimination or management of uncertainties at higher non-microscopic levels.

\section{Keywords}

Holism, Reductionism, Downward Causation, Wholeness, Incommensurability, Explicitness, Infinity

Subject Areas: Philosophy

\section{Introduction}

There was a time, the mechanical clock had just been perfected, when a new science arose espousing that this 
model could be applied to everything in the universe. Kepler wrote in 1605, "My aim is to show that the celestial machine is to be likened not to a divine organism but rather to a clockwork" [1]. Once the complete theory was elaborated by Newton, the solution of celestial mechanics—at that time mainly the solar system-became the ideal for any scientific approaches to the organization of matter. Even today's reductionists-for instance, the standard model of physics-consider the world like a swarm of non-dimensional, dot-shaped billiard balls interacting according to a set of fundamental laws and that science is nothing more than this, meaning that everything else can be constructed following this reductionist base. That the world as a whole is not a clockwork universe, nor behaves like a solar system or like billiard balls, has, of course, been pointed out all throughout history. One of the earliest cases is Mill's emergentism [2]. Two of the most striking formulations are Fodor's [3] “'immortal economist', who vainly tries to derive economic principles from a knowledge of physics and the distribution of physical qualities in space-time", and Ellis [4], who wrote, "It is inconceivable that ... fluctuations in the [early universe] ... can have had implicitly coded in them the future inevitability of the Mona Lisa, Nelson's victory at Trafalgar, and Einstein's 1905 theory of relativity”. There is clearly a problem. However, what exactly is the matter does not appear to be as trivial. Existing literature makes a strong effort to identify examples transcending reductionism. However, sometimes these topics are "over-discussed" which adds to the general confusion rather than clarifying the situation. Language is too weak to be able to give a clear account of such imbricated topics and their meandrous structures. The only way to succeed is to stick to the point: when, where, and in what sense it is possible to make reliable cognitive treatment-allowing reliable action, as science is just a part of this—at levels different from the basic reductionism.

Hence literature contains a whole battery of anti-reductionist approaches, including holism, downward (or top-down) causation, emergence, supervenience, several forms of uncertainty and sensitive dependence (chaos/ dissipative structures, the quantum physical arguments). Downward causation is one of the main pillars of our argumentation. For chaos and quantum physics some explanations will follow in chapter 2. First, as an introduction, a short discussion of the other approaches will be given, including an evaluation of the relevance for this paper. Detailed treatment as far as necessary and contributing to our quest will result from the main body of this text.

Holism [5] [6] best supports the general tendency developed here. There exists a global form of holism, including the whole of the universe [7]. This will be treated later. For the moment we consider the local form, which is the view that most of the items of our environment (physical, biological, chemical, technological, social, economic, mental, etc.) are stable complex organizational structures and their properties and behaviors should be cognitively treated as wholes. This often includes the view that entities function as wholes and that their functioning cannot be fully understood solely in terms of their smallest reductionist parts (adapted from [8]). The saying is that "the whole is more than the sum of its parts". But what does the word "sum" mean in this context? It will be shown that trivially everything more than one single particle, that is two or more particles, is holistic in our sense. The key notion in our approach is that of wholeness: something that can cognitively be seized as a whole, that is reliable and stable (at least under "normal" conditions) and following its own nonreductionist, global organizational laws or rules.

Emergence [9]-[11] is probably the most discussed of all anti-reductionist arguments. For our discussion the most adapted view is that of Laughlin ([12]; see also [13] [14]). His main argument is that there are physical systems whose properties are purely organizational and that at the level they are investigated, the underlying rules are rendered irrelevant, impotent by their principles of organization. In other words, such systems describe wholenesses. This terminology will be preferred considering that emergence is too close to reductionism suggesting a bottom-up approach (since something emerging has to come up from a lower level).

A very special case is supervenience (from the Latin supervenire, "come on top"). Fundamentally, it is the idea that there are laws and rules that supervene on structures at lower levels. Unfortunately, on the way to define this more properly, it became the following [15]: "A set of properties A supervenes upon another set B just in case no two things can differ with respect to A-properties without also differing with respect to their B-properties". In slogan form, "there cannot be an A-difference without a B-difference". This sounds more like a bottom-up procedure as in reductionism. In [16], it became completely this: "Supervenience is an ontological relation that is used to describe cases where ... the lower-level properties of a system determine its higher level properties”. Given this confusion, the term will not be used here at all. In any case, supervenience only involves very few aspects not already covered by downward causation, holism and emergence. Should the term be needed, a more adapted expression will be used which is "supersedience" from superseding (from the Latin su- 
persedere, "sit on top").

The existing discussion is almost completely limited to working out anti-reductionist scientific examples. The contribution of this paper is, by contrast, to give a global picture about everything that exists and how it integrates into the present debate, from elementary particles to cosmology, including all objects of macroscopic science and even of everyday life. Surprisingly, this can be made in a rather simple description and allows one to explain under which logical conditions reductionism and its counterparts can hold true and how the structures are articulated in between those two approaches. This text aims to include and discuss all logical possibilities of the organization of the world. Only a global view of all that is and its organizational articulation can create a sensible understanding.

\section{Fundamentals}

Making valiant efforts not to succumb to the all-is-physics oversimplification, it must be admitted that among our starting points, there is a type of physicalism. Although it is very different from reductionist physics, in my physical bones, so to speak, I feel that for any perceived reality, there must be a physical base. The upshot for avoiding overly simplistic interpretations is that this base is very complex, entangled and intermingled, with an infinity of patterns and structures. Once the highest imaginable complexity in this base is accepted, a sound conception can be given of how physicalist phenomena can generate adapted complexity-for example, in the brain-also involving infinity. Whatever is the point, it is probably safe to say that if one accepts such complexity, an epiphenomenal relationship exists between brain and mind. Such is the picture here: on one hand, a mindindependent physicalist reality, while on the other hand brain processes able to generate true representations in the mind, which are still physicalist. A brain-independent mind is still sometimes postulated nowadays. This belief may be correct, but it is an enormous speculative leap which will not be admitted in this paper and which is simply not needed.

A second point that has to be clearly stated is that almost the totality of science is making closed models. Indeed, all science selects a finite amount of elements in reality, combines them with finite rules of how they behave and interact. Once the model is chosen, it is closed against what is outside. This can be the previously mentioned swarm of elementary particles but is not limited to numerical models. It can also be, say, the mouse that is always running away from a cat. The notion of a closed model can also involve, though in a much more limited manner, other than natural sciences. It can be something as simple as how to open a door or a window. The opposite of closed models are open models among which include all systems showing sensitive dependence-involving uncertainty and bifurcations. A simple example of a bifurcation is a pencil standing on its top, of which if perfectly centered it cannot be said in which direction it will fall. A more evolved model is a ball running in a small valley that truly bifurcates into two valleys. Again, if all is perfectly centered (the shapes of the valleys, the position of the ball) and all outside influence is excluded you cannot say in which valley the ball will run. In reality things are never perfectly centered nor outside influences excluded, which are the factors that determine which of the two bifurcations will happen. But that is beyond what closed models can do. In such models it is not known what the small deviations are. Open models describe such open situations. Their virtue is to point out that in reality regions described by such models exist. Such regions are crucial for a philosopher in the sense that they are where all structural novelty is created. Open models have not one solution but at least two in the case of a simple bifurcation like the famous Lorenz equations [17], but any number of solutions can be imagined. One more step that is postulated here involves structural bifurcations happening on multidimensional structures and having as a result not just a behavior of an object but creating complete continuous structures that can be the base of downward causations. Nonetheless, open models are true models in the sense that they exclude the largest part (by far)—an infinity—of behaviors from their solutions. Chaos theory (nonlinear dynamic systems, nonequilibrium dissipative structures) is a simple case based on such principles (fractals in this case, as in the Lorenz equations). We go a bit quick as it can be supposed that philosophers are nowadays familiar with such concepts—namely, strange attractors or similar patterns (if not, see [18] [19]). What is less common is that the uncertainty of quantum mechanics (the Heisenberg principle) can also be reduced to a pencil balancing on its point according to the Nobel Prize winner Laughlin [20]. Indeed, already de Broglie (rediscovered later by Bohm) gave a causal formulation that amounts to that [21]. On another note, for this paper it is also important that there is a multitude of models not showing sensitive dependence and being robustly insensitive to details. There exists a minimal size (an $\varepsilon>0$ ) for which any perturbation smaller than this does not change the behavior of the system. Such structures, being a special case of closed models, shall be called regular models. By contrast 
to open models, it is precisely the characteristic of regular models that they show the same behavior independent of small influences from outside of the model. Complete formal descriptions of all these types of models can be found in [22].

A third point is the importance of several types of infinity [23]-[25]. These types of infinity, the infinitely small, the infinitely big, and the infinity "in between" (the one of sensitive dependence) are so fundamental that they will be at the core of the above-mentioned logical possibilities of the organization of the world. Elsewhere [23] it has been argued that agreeing on truth is only possible with good faith and intentionality, and furthermore, that any representation is only a partial view of finite aspects of the infinity of possible views. So everything we can say is always a partial point of view which, even if intentionally true, is incommensurable to other points of view. In our system incommensurability is the really important feature. It is directly linked to the notion of infinity, as it is defined by the fact that two items cannot be transformed into each other by a finite number of operations. Traditional critics of reductionism (see chapter 3) do not take into account incommensurability. Yet it is precisely this notion that will allow a more nuanced view of things.

\section{Reductionism in Trouble}

So let us first consider the anti-reductionist point of view. This will show as a first result that at least locally there is no point in trying to defend reductionism under any circumstances. Very strong arguments exist that rather than the smallest entities of reductionism, it is always the bigger constraints- the superseding structures - that form our world and are hence causally at the origin of things. Our logical structure starts with this issue, wholenesses that are determined by something else than a lowest level, reductionist bottom-up explanations of the world. We have to be conscious that this is for the beginning-like all discussion in that field—in a context of closed models, to keep things clean and clear. In such a framework, the two arguments that most resist a rigorous analysis are downward causation and local holism. For the latter, an extensive cluster of significant arguments will be given showing why a wholeness is more than the sum of its parts.

Remarkably, the field with the most examples for this argumentation is everyday life. No need to go to sophisticated physical or biological systems as is the custom to do in the literature. Namely, all the objects of our civilisation, almost everything that allows us survival and comfort, are all wholenesses caused by downward causation, showing behavior impossible to explain explicitly by the lowest level microscopic laws and not needing them for their explanation, like, say, a factory of canned food.

Here an explanation is needed about how causality is considered in this text. If you take deterministic causality, everything is causing everything else, which is, of course, neither a clarifying nor a really comforting argument. It is more adapted to define an efficient cause. The efficient cause is an event that creates a change of behavior of an object, system or any portion of reality. It is the reason why these are deviated from their inertial behavior that they would have shown without this cause. As Laughlin [26] writes, in the movies, Godzilla is the efficient cause of the destruction of Tokyo and not the laws of physics or of biochemistry. Seeing such things already gives the first example for a physical downward causation. It is this type of cause that is considered here, excluding the hypothetical mind-body downward causation that is at the origin of so much discussion [27]-[29]. As already explained, this paper has a different view on that.

As seen above for closed models, regular models are neither necessarily mathematical; they can namely also include predicates and language in general. In such a context, downward causation becomes evident. Wholenesses like a chair, a table, other types of furniture, curtains, crockery, food, drinks, household machines, computers, cellular phones, cars, and so on—all achievements of any form of human technology—are caused by either artisanal or industrial production facilities and their supply chains which are the efficient causes of all these items. The wood in a table would still grow in a forest (its inertial state) if it had not been cut and transformed into a table by human downward causation. That is the cause of the table and not the table's elementary particles interacting and associating to form a table. The particles here are always under control of higher, superseding levels of organization, the efficient causes. The position of any elementary particle does not matter. In situations where they could matter, e.g., for the formation of molecules, they are there always in masses and if it is not one particle that is integrated there are millions of others around that can be selected. That is the principle of organization of our whole world; it is based on eliminating uncertainty by downward causation, something profoundly opposite to reductionism, where the systems are supposed to work thanks to the certainty of the precise evolution of the elementary particles. The tree is a good example because one of the factors that determine its growth 
is its DNA, and there again the precise position of molecules does not matter. Be it for duplicating DNA, for creating messenger molecules or for directing the synthesis of proteins-in all these cases, position does not matter. Again, if it is not one molecule of the needed type that is integrated, it is another. Further factors influencing the tree are the quality of the soil, weather, climate, accessibility of light and many more. All these are downward causations. Note that the concept of what is downward is defined by organizational levels, not by physical size. The Egyptian pyramids are much bigger than their efficient "downward" causes. A tree might be attacked and even killed by microscopic parasites, which although small belong organizationally to the higher level of epidemics in an ecosystem. This clearly establishes that the real world is not a clockwork universe or a swarm of billiard balls, not even one resembling a planetary system. Reality has a completely different structure based on supersedience that controls uncertainty and makes things independent of the certainty of reductionist bottom-up parameters. One aspect is the large numbers. Just below, a few examples will be given showing how with large numbers properties appear that cannot be reduced to microscopic laws. It is also that there are so many that one can remove from any macroscopic object, say, a million elementary particles (e.g., atoms) without changing any properties of the wholeness. It is not at all like a mechanical clock that instantly stops working as soon as you take out a wheel or a screw. Another difference is that there are layers in scale, several levels of wholenesses, each towered on top of the smaller one. Such is the very way downward causation appears: upper layers supersedingly controlling lower ones. The issue is not how to describe every element precisely, but rather to find out what is limiting and channeling - that is, what is organizing supersediently the uncertainty of large quantities of elements.

It is probably a good idea to make a connection between downward causation and the second topic of this chapter which is holism. This topic will be considered under a great number of aspects. We will call "critical plus" the famous feature that the whole has more than the sum of its parts and which can already now be described in a surprising way: wholenesses are always determined by bigger efficient causes. So what makes them what they are is not their reductionist composition but the downward causation of their environment. All the wholenesses mentioned above and conditioned by downward causation are also wholenesses in the sense of holism. Wholeness is created and determined by boundary conditions, external shaping (e.g., all manmade objects), global parameters such as temperature and pressure, or robust chemical processes as in biochemistry around DNA. In such a context, downward causation becomes the first argument for holism. The critical plus comes in this case from all the influence of a wholeness's superseding structures. This argument holds as the outside influence is clearly not in the reductionist base of a wholeness.

The most convincing argument in the literature, giving a very clear explanation of the critical plus, comes from Laughlin [30]. He states that the claim that for collective principles there is always a deductive path from the microscopic is "clearly incorrect." In the example of temperature, the microscopic explanation needs a non-deductive logical step called "the postulate of equal a priori probability." Furthermore, the deductive explanations of the Josephson and von Klitzing effects always have an "intuitively obvious" step in which the relevant macroscopic information is hidden. Moreover, there are cases in which you can introduce a fictitious law of motion and after calculation you arrive at the correct result [31]. Also, according to Healey [32], the basic laws of statistical mechanics do not determine the principles of thermodynamics "without some assumptions (however weak)". Likewise, Cushing [33] cites for the Quantum Field Theory a list of such assumptions, including negative-energy sea of electrons, discarding of infinite self-energies and vacuum polarizations, and permanently confined quarks (among many others). This makes one think that reductionism is probably also in many other cases not as straightforward as its proponents would like to see it. After all, the burden of proof for each claim should be on the shoulders of the reductionists. There is also the complication that Quine [34] has shown that linguistically reductionism is not logically possible in a consistent way, as well as the notorious failure of Carnap when he tried to build a reliable scientific language based only on reductionist syntax [35] and had to reintroduce semantics [36], which involves among other wholenesses targeted by the meaning of words.

The next group of claims justifying the critical plus in the literature comes from the large number of elements and from the complexity of organization in multiple levels. Laughlin [12] insists that there is a rich range of "protected" properties that are insensitive to "microscopics" (also [13] [14]). The behavior of these properties is well understood through high-level principles while inexplicable in fundamental physical terms. They are subject to the "essential role played by higher organizing principles" [37]. There are "yet undiscovered organizational principles" [38], which we would call superseding laws. The issue is not that the underlying rules are wrong or irrelevant but they are rendered impotent by principles of organization [39], e.g., crystals sponta- 
neously acquire a property not present in the underlying rules themselves [40]. The universe can only be organized by "collective principles of organization" [41]. These principles are transcendent in that they would continue to hold even if the essentials were changed slightly [42]. Laws do not cause organization but organization causes laws. The world is not constructionist founded on the basic laws: at each level of complexity entirely new properties appear [43]. Very simple examples of such properties are the rigidity or elasticity of solids or the wetness of water. According to Bishop [19] [44], fluid systems acting as a whole may produce collective effects that are not reducible to a summation of the trajectories and subelements composing the system. Anderson [45], mini-bibliography on that topic in reference 3 of [45] and Laughlin [12] strongly insist on the notion of symmetry breaking as origin of higher organizational rules. This refers to the idea that the infinite symmetries of the continuum are reduced and thus "broken" by lower symmetries that appear at a higher level; for example, the "atoms in a crystal have a clear symmetry, preferred positions, even though there was nothing preferred about these positions before the crystal formed" [46]. Other examples given are magnetism, superconductivity, phases (liquid, solid, gaseous) and some stranger new phases like supersolids [47] and superfluids [48]. Laughlin claims this principle even for explaining the thought experiment of Schrödinger's cat, symmetry breaking being required for the apparatus to make a non-controversial sense [49]. This also supports our claim that going from one single particle to a pair already constitutes a wholeness, as this is clearly a symmetry breaking. In all this, the critical plus consists of the laws at high levels created by such organizational phenomena. However, it is a somehow odd situation for holists since the reductionist origin of such phenomena cannot be falsified with absolute certitude. And it is equally odd for reductionists as they cannot calculate more than ten particles, even with the most powerful computer. For explaining the size of molecules and the length of chemical links, they have to make approximations [50]. Yet approximations are a tricky thing from a philosophical point of view. First, you never know if the approximations made are accurate. All you know is that they work in some cases, but you might be in a case of sensitive dependence in which the result will be completely wrong however small the error of the approximations is. Furthermore, you construct such approximations knowing what you want to proof and adjust them until you obtain what you want to know. Can such a thing really be called a proof? What is clear is that in this paragraph there are wholenesses as well as new properties that fit only these wholenesses and that can be completely understood and described at a given superseding level of observation without knowing anything about microscopics. We have at least methodological holism [51].

It may be a good thing to continue on a rather philosophical tone, as the available principles for physical argumentation have been exhausted. The main philosophical argument is the one of form, the famous "Gestalt." It deals with the transition of individual entities, say, points, to a continuous form, such as a line or a circle. In that process, the points are melted away into an upper dimension. The small interstices that always exist no matter the closeness of the points are disappearing. Any form in the examples of wholenesses mentioned above does exactly this. The critical plus in this case would be this melting down, this smoothening of the interstitial spaces. Indeed, this argument stands firmly as the necessary element, for the holistic description of wholenesses is precisely the shape of things in a higher dimension. Consider an Impressionist painting (Renoir or Monet mentioned by [52]). The daubs of paint are randomly shaped and make no sense. It is only when looked at from a sufficient distance that the form in the painting appears. The irregularities and imperfections of the individual brush strokes disappear. What is more, the wholeness of such pictures is completely independent from its reductionist explanation. It could be painted on stone with another type of colors, and the wholeness would be exactly the same. A digital photograph follows the same scheme. It can be looked at on different screens, printed on paper or any other medium, stored on a memory card or stick, or on a hard disk, etc., but the wholeness of the picture goes on being the same and is completely independent of its reductionist base. In these examples the critical plus of form is obvious. Whether this is also the case in all previously mentioned physical systems can be a matter of dispute. The properties of a crystal depend on its precise reductionist chemical composition, which can only in specific cases be switched without changing the characteristics of the crystal. However, philosophically the argument of form can be maintained also in these cases. There is always some smoothening, some wiping away of details that allows the wholeness to be seen. It is really the wholeness, the shape or form that makes the essential critical plus. It's something that can stay in brains, on pictures or in other means for conservation of forms, long after the particles forming them have disappeared. Again, even two elementary particles are a form. The second opens up a new dimension that was not at all in one single particle, the dimension of a line. A third particle either continues the line or again opens up another dimension, the one of tridimensional space. And so on: every new particle adds something to the preceding pattern. From the fact that we cannot say when macros- 
copic properties start-for instance, how many water molecules we need to get wetness-it has to be concluded that whatever is more than a single particle or molecule, starts to be a pattern, a shape, a form, a Gestalt; and therefore constitutes a primitive type of wholeness containing a critical plus.

A step further towards an indubitable critical plus is given when it comes to signs, symbols and language. In these cases, there is an additional element which is convention. Support independency is given as before (meaning that some support is always needed, but it does not matter which type). But in this case, to understand we need to know something more than what is in the physical support. The letter A is a form, but it is not clear to which sound it corresponds unless you know it. This leads to human brains but not only. Also computer code is a language, with the convention built into microprocessors that know how to interpret, that is, know what to do with each instruction composed of bits- $1 \mathrm{~s}$ and $0 \mathrm{~s}$ - again support-independent. Yet this last example ultimately has also been conceived by human brains. In any case, it is important to realize that language is-by conventions - at the origin of the largest part (by far) of downward causations one is confronted with. All that is not natural science goes into that category: the laws of the different legal systems, contracts, conventions on how to behave in society, political attitudes, fashion, and psychological constraints of individuals about how one should be and should behave. All these, although often in conflict, make individuals act in a certain manner determined by the efficient causes of the hereby created supersedient downward causations. For the sake of completeness, it should be mentioned that in this domain, the one of human brains, downward causation is not always efficient. There is a great deal of phenomena, like by the way also in other domains, that cannot be controlled, but have to be dealt with as well as can be. The royal example is emotions, which are working in a completely different way but are fundamental for the efficiency of brains.

Even more ludicrous for reductionism is the famous affirmation of Heraclitus that you cannot twice step into the same river. What makes the wholeness of a river? Certainly not the water molecules that flow through it as they are constantly replaced by others. Nor its banks: although they give the shape of the river, they are not the river. Imagine further a car belonging to Mr. Miller and that you change all the parts it is composed of and replace them by identical ones. Is this still the car of Mr. Miller? It is also well known that living beings change all their atoms several times during their lives. For a human this has been estimated to happen every nine years [53]. Or take fossilization, what is dug out and put in museums is not at all bones in their initial composition, but mineralized copies of them. The carbon has transformed into its mineral form calcium carbonate. Again what remains is the form. Along the same lines, imagine that your kitchen table would change all its atoms for others of the same type. Would it still be your kitchen table? In a reductionist approach, the upshot of this is that Mr. Miller is not only not sure if his car is still his car, in addition, he is neither sure if he is still Mr. Miller. It has been calculated that atoms move so much that everyone of us is composed by about a billion atoms that belonged once to Shakespeare, or Beethoven, or Buddha, or Genghis Khan [54]. The world follows more the "pantarei" (everything flows) of, again, Heraclitus than a clockwork universe. It is probably safe to say that here is the final proof of what makes the world is wholenesses. Mr. Miller can be at ease: the wholeness of his car is not in doubt, nor is his own body. Moreover, this gives a whole group of new clear explanations of the critical plus. Living beings and many other items such as lakes, oceans and clouds are doubtlessly not created by reductionist causality, but by form, shapes and wholeness - and we come back to downward causation that creates and stabilizes all that (for some time at least).

One idea that has also to be considered, is what would be if the atoms of our already frequently cited table would really permanently change, flowing through the table like water in a river. In such a case it would no more be the atoms that make the table but energy fields that constantly attract particles and make them flow through it. Maybe the "pantarei" is to be taken as literally as that. This would solve the problem how with wave functions whose probabilities are distributed over the whole universe there can be stable macroscopic objects. The objects would be fields of energy that attract the wave functions in such a way that there is always one in the place needed. But then the object would clearly no more be the particles, but the energy fields that give it its form. Such fields would be continuous, and thus infinity-based. One logical possibility to get the critical plus is to suppose that there are no elementary particles, but smaller and smaller entities without limit if you go down in scale. Even string theorists consider that strings, which are already tremendously small (at the size of the Planck length, which is extremely small), might be composed of even smaller structures [55]. In this case the critical plus would be formed by these ever smaller particles. There would be no way to say that an object is formed by elementary particles as there would be none. The above energy field would be a bit similar, and with continuity it would always also be in the interstices where the particles are not and by this form the critical plus. As an in- 
finity-based field (continuity), it would define the form of the object. Is this a sense that can be given to Aristotle's "entelechy"? The word is used by some modern philosophers precisely to justify a critical plus. In the case of life, it's the vitalists that have such claims, something like an "élan vital" [56].

Regarding field theories, it has to be added that even main science field theories, such as the Quantum Field Theory or the gravity fields of General Relativity include possibilities to produce the critical plus. Being continuous they all potentially allow perturbations at any scale, namely, there is no limit to the smallness of such. Even if I do not know of any attempt to interpret them in this way, they include exactly the same possibilities to have infinitely small structures, like the infinitely small particles mentioned above. All of them clearly are potentially anti-reductionist. Another logical possibility regarding field theories has to be mentioned. They could have the property to allow spontaneous perturbations, very small warps or ripples which could-according to their environment-develop and get bigger with time, until they reach a stage where they influence observable things. It is not a possibility I would go for, but it has to be mentioned. Actually, the existence of spontaneous phenomena at low scale in general is an issue that would solve many philosophical problems in a highly convenient way.

\section{A Global Vista: Options for a Better Philosophy}

Does this elaborated assemblage of shortcomings for reductionism resolve the matter? Not quite. There are elements to come, another point of view, which will again completely upend the conclusions made so far. In spite of the closed model's quasi-monopoly in all scientific, philosophical and cognitive approaches, with supposedly nobody being aware of it, there are alternatives. In many cases, the world outside the model cannot be neglected. Is the solution to replace closed models by open models, sensitive dependence and infinity-based approaches? Unfortunately, things do not plainly unravel like that. Although there is a bit of all these elements, it happens in a very particular way.

But before going to this here we have to slow down a bit and first give the promised global view of what there is. Starting with the types of wholenesses-already the smallest, elementary particles, needs some remarks. It has been shown in what sense two particles form a wholeness. Together with Laughlin [57], it is here claimed that even a single electron (and, by the way, a fortiori also two, three, and more of them consistently with what was already said) is an organizational phenomenon, which means a wholeness in our terminology in the sense that such isolation does not occur naturally but only by use of a very big experimental apparatus exerting downward causation. What is more, as a matter of fact, there is no reason why an electron (or any other particle) should be exactly identical to another. This claim is one illustration of our first door of entrance for infinity: the already evoked particles or field structures being smaller and smaller without limit. If going up the scale, in the micro-molecular world, chemistry also treats wholenesses, though reductionism has some merit there for giving the models of atoms and the chemical bonds. But the precise behavior of chemical reactions has always to be worked out empirically on wholenesses involving a very large number of molecules. The next items are already living beings and-next group-all creations involving contributions by living beings, including ecosystems, societies or the wholenesses of chapter 3, including science or the objects of humans' daily use. Furthermore, some forms on our planet are not created by living beings, for example, lakes, valleys, mountains or continents (created by glaciers, tectonic push, volcanic activity or erosion). The last step is cosmology with all the celestial bodies, from the smallest meteoritic dust to galactic superclusters. For the last group, it is interesting to know that its elements are created in two ways, by the big bang that everybody knows and which supposedly created only hydrogen, helium and really very little dash of lithium [58], but also by what I call the "small bangs," the supernovas that create and disperse in the universe all the rest of higher elements [59]. That is the reason why the universe is 14 billion years old and the Earth only a bit more than 4 billion. There was need for some big stars to burn out and explode before our solar system could be created. Concerning the universe, in principle the last step in our list, it is interesting to know that its four dimensions (three of space plus time) are supposed to be curved and to close on themselves like the line of a circle. But as the second dimension is needed for the circle to be possible, this means that the universe requests at least a fifth dimension. What is this made of? There might be other things out there, other universes or other forms of worlds. Some speak of macro-universes. This point is a second possibility to introduce infinity as the world of the worlds might be infinite. We just do not know and cannot know.

That makes only six types of wholenesses that all follow the principles of closed and regular models. They have all a certain stability, are insensitive to small perturbations and their behavior can be described superse- 
dingly, independently of any lowest level's microscopic laws. However, it is crucial to state that their stability notwithstanding, as with all wholenesses they are cognitively useful to the highest degree and ontologically significant, but they are not absolute-nothing is. For instance, they all have a beginning, an evolution during their existence and an end. To discuss the last point first, some just slowly fade away (a melting glacier, an eroded mountain, living organisms that become too old and eventually just stop living). Or they end with events being above the threshold of insensitivity of the regular model of a wholeness: a meteor falling on a mountain, a part of rainforest destroyed by bulldozers, the Earth "eaten" by the inflating sun at the end of her life, the kitchen table that is getting too old and ends up in the garbage where it is destroyed. Secondly, they all evolve in some way. Even crystals are aging, although this aspect is the most pronounced for living beings which-before aging - grow and sometimes undergo drastic changes, e.g., all animals that have larvae. But all these latter changes come from inside the wholeness and are part of the model (regular models organized by DNA). It is also part of the model that some of these wholenesses adapt to downward causations like the discussed case of a tree.

To discuss the beginning of these wholenesses, we have to come back to sensitive dependence which introduces the third type of infinity, in the form of the continuity of space-time on which such dependence is based. A list can be made as well of the critical domains related to this point which are the items where the fundamentally new is created. Only the bifurcations of sensitive dependence can create real novelty, meaning incommensurability. It is these sensitive dependent domains that are directly or indirectly at the origin of all the above wholenesses. It might seem surprising but after long reflection I privately think that in nature there are only five of them: atmospheres of celestial bodies (including weather and climate), liquids or fluids inside of celestial bodies, brains, evolution and cosmological systems (small bangs, the big bang and all resulting celestial bodies). All these domains are dynamically under the influence of sensitive dependence. They cannot be described by a closed regular model, but need open models, as any influence from outside the model can change their behavior. It is interesting to observe that the first two domains and their effects stay inside of a superseding wholeness. That means that even structures creating incommensurability are doing this inside domains of reality limited by downward causation they cannot overcome. Weather and climate as atmospheric phenomena influence heavily what happens on Earth (e.g., the ecosystems and species that live in them, ice ages and erosion), but have no impact on anything outside the atmosphere. The liquids inside the Earth are responsible for important effects on the surface: everything related to volcanic activities or plate tectonics caused by convection of these liquids that create mountains and the movements of the continents. Nevertheless, these phenomena again stay limited to the wholeness which they are part of, that is, our planet. For brains and evolution the situation is similar most of the time. They are, however, related to life, of which one characteristic is that it sometimes does go out of its surrounding wholeness (humans have even left the planet). Life has on top the capacity to arrange things to its own advantage. For evolution, as seen, DNA is the base of the regular models of living beings, although it must be stated that we have not the slightest idea precisely how the information on the DNA ends up really making differences in the living being. In any case, it happens-relatively often actually-that the copies of DNA have errors, most of them with no influence, but sometimes they change the organism, which is then tested by the next higher wholeness, the ecosystem, that eliminates bad mutations and keeps positive ones for later generations. About the chaotic patterns of this process, see [60]. All this is part of evolution for which some also claim the need for additional explanations to pure Darwinism (e.g., the already mentioned vitalists). Brains are even better for life. They make the profit immediately available to the organism-no need to wait for the next generation. Like with DNA, we have no precise understanding of even the organizational principles of brains. It has been held elsewhere that brains might be sensitive dependent devices [61]. The idea is that they use very small fluctuations, outside of the brain's sensitive dependent regions, to create by these regions structural novelty incommensurable with earlier states of the brain and allow adaptation to new requirements-also incommensurable-coming from the outside of the organism. The wholeness of the organism is regular in the sense that it can eliminate these fluctuations in the event they are not creating profitable brain structures. This includes all humans and their brains as open models, the open brains, which if considered as sensitive dependent can explain all the valued things like creativity, responsibility, intentions and free will. Additionally, all the scientific systems discussed in chapter 3 are in fact portions of reality prepared by causal chains coming from human brains in order to obtain the desired results. This is also the case for the already repeatedly cited objects of daily use. A smartphone, say, has its main efficient causes in probably thousands of brains. Likewise, the massive presence of sensitive dependent open brains explains the limitations for prediction and the increased difficulty of finding agreements in other than natural sciences like psychology, sociology, economics and politics. As mentioned, in 
these fields downward causations are only of limited efficiency. They work a bit but, for example, often only on a part of the open brains. In any case, Fodor's "immortal economist” can be relaxed, economic principles can in such an approach at best be derived supersediently. And there is no other way that makes sense; even reductionists have to accept sensitive dependent regions. Regarding the last critical domain, it should be noted that the celestial systems resulting from the different bangs are also all sensitive dependent, as according to a theorem of Poincaré all nonlinear multi-body systems with more than two bodies are chaotic because their equations cannot be integrated explicitly [62]. More importantly, it is extremely surprising how easily science accepts that the big bang produced ordered structure, rather than a homogeneous nothing as should have happened according to thermodynamic laws. Remember the big bang produces mainly gases (hydrogen and helium). Such have the characteristic to occupy any available volume homogenously. In no way should they agglomerate, not even under gravitation or in very large volumes. The current official explanation [4] is that there were "truly random quantum fluctuations in the inflation field during the inflationary era" and that this led to formation of cosmological objects rather than to homogeneity [63]. Must this be understood as one of the above spontaneous fluctuations? Or is it meant to be just some random favorable combination of micro-events inside the reductionist laws of nature? It was always my opinion that our world is too beautifully and efficiently structured to be explained by random events. Some theories explain this issue with "dark matter" [64]. But this is really in its beginning; we do not even know what dark matter is and whether it exists. As Padmanabhan states [65], "clearly ... we do not yet have a satisfactory theory to explain the observed structures in the universe" (and there has not been any substantial progress since). The problem is the same with the small bangs. With a random event we would get nothing, but we have something - with even very nice structures. It is striking that we have now five topics in which, according to us, there is a strong chance that new organizational principles have yet to be discovered that will be anti-reductionist: DNA, evolution, brains, small and big bangs, and the "yet undiscovered higher organizational principles" of chapter 3 . According to the position of reductionism, all that results from the basic entities and the basic laws. The rest is considered to be purely random, as a type of self-organization not needing any other organizational principles. In any case, it must be stated that between the two extremes of claiming everything purely random and of claiming nothing random, there is enough space for intermediate positions, like the one suggesting that there are one or more yet unknown features in the very nature of matter that make forms of organization more likely than complete disorganization, a type of DNA for matter, but much weaker in its determination. This would not be something that totally determines things, but that just provides some guidance, helping matter to get into an organization making sense rather than into a senseless, microscopically chaotic nothing, as it should according to the laws of entropy. Whatever there is about this, it should be considered. It is a logical possibility and must therefore be mentioned following the declared ambition of this paper.

These two inventories contain all the elements to resume the discussion of reductionism at the necessary higher level. If supposing that ultimate elementary particles exist, the upshot of all this is that if we enlarge the closed and regular models in space, but more importantly in time, sooner or later there will come the moment when they no longer describe wholenesses but the different types of sensitive dependence appear in these enlarged closed models in a way that they are no longer regular. Moreover, if taking the finite universe of the official big bang theory, the number of particles must be finite. It is hence possible to make a closed model of the whole universe that includes all sensitive dependent regions and their open models. All particles able to influence the bifurcations of the sensitive dependent portions are part of this model. To that has to be added that all the superstructures making the downward causations are themselves composed of elementary particles, and are therefore part of this closed model. At once it seems that reductionism is back, although in this case also, as earlier requested, it is up to the reductionists to prove that claim (including an explanation of how all the arguments of chapter 3 are finally reductionist), which is, of course, impossible. We do not know and we will never know if such a closed model could be held true. But it is a logical and ontological possibility, although the resulting reductionism is in this case only in principle. Seen from this aspect, open models and the infinity of the continuous space-time make possible a consistent form of reductionism in which sensitive dependence supports reductionism and not the contrary, as it is often considered. This is a key result showing that a sensible conception exists which even gives an ontological reality to reductionism.

Such is the reductionist point of view as far as we can take it at its best. Nonetheless, there is also an anti-reductionist point of view on the same infinity conditions. To start this discussion, first take the second infinity, the infinitely big world, which avoids reductionism in the sense that by the definition of closed models no such model, as the one above that revived reductionism, can be given because we have an infinity of particles 
and an infinite space-time. But such a universe is still holistic as the chain of ever bigger and bigger downward causations is infinite and because of this infinity cannot be reduced further, even in principle, to reductionist particles. Conversely, reductionists could claim that even the infinite case is just a limit with more and more particles. All steps towards this limit being reductionist, the limit must have the same property. We have a type of stalemate situation. This global form of holism announced earlier is at the same time reductionist and holistic, the two of which become indistinguishable. We must consequently conclude according to Leibnitz that we have an identity of indiscernibles. It is not that the question has no sense, but only that with these conditions a world would be naturally both reductionist and holistic. Yet there is a cognitive difference between the two that must be highlighted here. Because any calculation has necessarily limited precision, no global reductionist model allows the deduction of the macroscopic properties explicitly as soon as there is some sensitive dependence at any lower level. And cognition needs explicitness to be able to act. Wholenesses, by contrast, allow explicit deductions as they deal only with the regular models that have already eliminated sensitive dependence. Global holism, however, might involve as the ultimate level sensitive dependence, e.g., the gravitational interaction of multi-body systems. In this case reductionism and holism are also cognitively reconciled again.

Coming back to the context with a finite number of particles, the anti-reductionist point of view must stay valid for the concerned portions of reality. The wholenesses stay wholenesses, open models stay open models, no matter how much the system border is enlarged. What's more, the sensitive dependence is not necessarily at the level of reductionist elemental particles, but can as well be formed by the combination of wholenesses, analogously to many artificial chaotic systems, such as the magnetic pendulum. Also, for instance, in brains there is no indication that if there is sensitive dependence this would be at the level of elemental particles. Besides that, in this perspective the infinity of the continuous space-time also supports anti-reductionism in another way. The here present enlargement of open models, even if finite, contains due to continuity an infinity of possible structures, each of which having the potential to change the bifurcations of the open models. The simplest is a single particle that has in a continuous space an infinity of possible positions, each of which can with enough complex sensitive dependent regions produce a different incommensurable downward causating superstructure through a (probably very complicated) net of structural bifurcations. Incommensurability is really the key notion here. Such an approach deals not with mechanic-like processes to be afraid of, but the concerned structures really create-potentially an infinity of-structural novelties, that is, the incommensurabilities of feelings, intentionality and real intelligence. Or take the example of a joke: it is highly probable that its understanding needs the true creativity of incommensurability. In a physicalist universe, by definition such true creativity can only come from sensitive dependence, and this property of open models must stay valid whatever the context. This is the key point: explicit structures issued from such infinity are ontologically not explicitly differentiable from structures out of other "clearer" uncertainties, such as the ones that are probabilistic, noncausal or nondeterministic (the latter as seen with the spontaneous fluctuation above). More precisely, with sensitive dependence this applies for any finite nonzero level of observation or calculation (which is called in mathematics, for any $\varepsilon>0$ ). The Mona Lisa can continue to smile: she is a downward causation by Da Vinci and thanks to the multiple incommensurabilities involved, the explicit effects coming from the bottom-up influences is the same as noncausality. The preceding arguments show that even in the present case of a finite space-time, it is possible-thanks to its continuity - to have an incommensurable infinity of downward causations. By such an argument, the open models seen from this different angle create the anti-reductionist interpretation of the finite case. Such results, which may be surprising for some readers, are possible thanks to the mathematical properties of infinity in which there is a bijection between any two infinities, even if one is a real subset of the other. The systems with a finite number of particles but with an infinity of points in the continuum are the subsets of the case discussed above with a real infinity (of particles and of space-time). In principle, there is no ontological difference! We must therefore again have a stalemate situation. This form of holism, which is also global, is at the same time reductionist and holistic, the two of which become indistinguishable. Consequently, it must again be concluded that we have here an identity of indiscernibles. This conclusion is not a problem but rather constitutes a solution. It is not an epistemological oddity. On the contrary, it is, like all other indiscernibles, a natural property of things as soon as real infinities in the world are accepted. Moreover, with regard to explicit deductions, the situation is again the same as above. Such reductionism can only be in principle and does not allow explicitness at higher levels, which wholenesses do!

Incidentally, it is not even necessary to take a whole finite universe. Any subset having enough complicated sensitive dependence and surrounded by continuous space and having enough particles to create downward cau- 
sation, is adequate. For instance, this is the case for the human society with its downward causations derived from language and its other conventions, with all the brains and social and political rules. All this is complex enough to potentially create an infinity of behaviors and real incommensurability, in new superseding structures (new legal laws, fashions, etc.). Furthermore, the same applies to cosmology which, being sensitive dependent, creates ever new configurations, and, thanks to the continuity of space-time, an infinity of them is also possible in this case, even in a finite universe. All these conclusions are emphasized by the intuitively clear fact that none of these systems will ever be in exactly the same state twice, no matter the amount of time that passes.

\section{Conclusions on Reductionism and Infinity}

Let's start conclusions with the most favorable case for anti-reductionism. If we admit the infinitely small and suppose that there is no lower limit for finding ever smaller entities or structures, reductionism has no sense. Furthermore, a clear sense can be given to the critical plus. It is the contribution of this infinite smallness to any construction based on supposed ultimate particles. Any existing and future field theory is, through its infinite smallness of the continuum, at least theoretically and potentially part of this case.

The other extreme is the case of the infinitely big. As this infinity can be approached by both reductionism and its counterpart, we lose any possibility of distinguishing the two apparently contradictory properties. They become one in infinity by using the principle of identity by indiscernibility.

In between is the infinitely small of sensitive dependence, which fundamentally relates to continuity of spacetime. Be it uncertainty of quantum physics, the one of chaos or other even more complex sensitive dependent structures, that is where the fundamentally new of other classes of commensurability is created. Nevertheless, becoming conscious of that possibility to generate fundamental novelty—incommensurability—might help to accept reductionism, if it comes to the worst of having none of the two preceding cases. Ontologically, the situation is analogous to the infinitely big, with the difference that in this case the infinity is tacitly accepted by most scientists and can thus be considered as a highly valid hypothesis. As seen, this case also shows an identity by indiscernibility, where downward causation applies as much as reductionism. With continuous space-time, metaphysically it does not matter if the universe is finite or infinitely big. The potential mathematical structures are expected to be the same, according to the mathematical properties of infinities set forth above.

It must be also observed that from a practical point of view complexity entails methodological holism, even without any form of infinity (such as sensitive dependence turning out not to be relevant). We cannot know enough. Even in this case, cognition must be based on management of uncertainty. Methodological reductionism, certainty of the individual positions or of other properties, does not make sense in no context. As Popper once stated, the computer that calculates such a world would have to be at least as big as the world itself. The situation is even worse: if sensitive dependent regions exist, there is no way to calculate macroscopic properties, whatever the size of the computer. The decisive criterion is that with sensitive dependence no macroscopic result can be obtained explicitly. All that remains an issue for the macroscopic world is the question about reductionism in principle.

Another favorable case for anti-reductionism is the existence of anti-reductionist brakes, of higher organizational principles that really are more than the sum of their parts. Examples for this have been given in chapter 3, but for generalization, formal explanations of the "yet undiscovered higher organizational principles" have to be found. Moreover, the question must be asked whether random combination according to a few fundamental laws is enough to explain the beautiful organizational structures observed at any level of scale. We have identified five fields where such lacking of-anti-reductionist—explanation applies. Should there not be something like the DNA of matter, organizational information hidden in the structure of matter that guides the processes without determining them completely, that pushes matter to form something rather than nothing? This need seems to be particularly pronounced in the cases of the small bangs and the big bang.

In any case, it has been shown that from a philosophical point of view there is—even ontologically—clearly more than only reductionism as in forms and support-independent information (language, etc.). Also in practical life, downward causation and wholenesses are the principles we can really work with. Even if reductionism would be the case, its interest would mainly be for studies of the world's properties at a very small scale, which will certainly prove to be quite useful but due to limits of calculation restricted to a very narrow range of phenomena. The general paradigm is therefore not the certainty of a clockwork, but the uncertainty of highly complex structures that are supersedingly controlled, or at least limited or directed from top down as good and, 
wherever it is possible, independently of the certainty of the precise properties of every ultimate particle, if such exist. Finally, it is important to point out that such superseding control is not always possible. We are confronted all the time with structures coming from sensitive dependence which are not controllable at the level we would like them to be. We just have to deal with them the best we can. The most important examples for this are many of the behaviors of human brains.

\section{References}

[1] Boorstin, D.J. (1985) The Discoverers. Vintage, New York, 71.

[2] Mill, J.S. (1843) System of Logic. 8th Edition of 1872 Longmans, Green, Reader, London, and Dyer, London, 427-430.

[3] Fodor, J. (1974) Special Sciences. Synthese, 28, 97-115.

[4] Ellis, G.F.R. (2009) Top-Down Causation and the Human Brain. In: Murphy, N., Ellis, G.F.R. and O’Connor, T., Eds., Downward Causation and the Neurobiology of Free Will, Springer Berlin, 75.

[5] Healey, Ri. (2009) Holism and Nonseparability in Physics. In: Zalta, N., Ed., The Stanford Encyclopedia of Philosophy. http://plato.stanford.edu/archives/spr2009/entries/ physics-holism/.

[6] Oshry, B. (2008) Seeing Systems: Unlocking the Mysteries of Organizational Life. Berrett-Koehler, Oakland.

[7] Bohm, D. (1980) Wholeness and the Implicate Order. ARK Edition, London.

[8] Wikipedia (2015) Holism. http://en.wikipedia.org/wiki/Holism.

[9] O’Connor, T. and Wong, H.Y. (2012) Emergent Properties. In: Zalta, N., Ed., The Stanford Encyclopedia of Philosophy. http://plato.stanford.edu/archives/spr2012/entries/ properties-emergent/.

[10] Corradini, A. and O’Connor, T., Eds. (2010) Emergence in Science and Philosophy. Routledge, New York

[11] Clayton, P. and Paul, D., Eds. (2006) The Re-Emergence of Emergence. Oxford University Press, Oxford.

[12] Laughlin, R.B. (2005) A Different Universe: Reinventing Physics from the Bottom Down. Basic, New York.

[13] Laughlin, R.B. and Pines, D. (2000) The Theory of Everything. Proceedings of the National Academy of Sciences, 97, 28-31.

[14] Laughlin, R.B., Pines, D., Schmalian, J., Stojkovic, B.P. and Wolynes, P. (2000) The Middle Way. Proceedings of the National Academy of Sciences, 97, 32-37.

[15] McLaughlin, B. and Karen, B. (2014) Supervenience. In: Zalta, N., Ed., The Stanford Encyclopedia of Philosophy. http://plato.stanford.edu/archives/spr2014/ entries/supervenience/.

[16] Wikipedia. Supervenience. http://en.wikipedia.org/wiki/Supervenience

[17] Sparrow, C. (1982) The Lorenz Equations: Bifurcations, Chaos, and Strange Attractors. Springer, New York. http://dx.doi.org/10.1007/978-1-4612-5767-7

[18] Smith, L.A. (2007) Chaos: A Very Short Introduction. Oxford University Press, Oxford. http://dx.doi.org/10.1093/actrade/9780192853783.001.0001

[19] Bishop, R.C. (2004) Nonequilibrium Statistical Mechanics Brussels-Austin Style. Studies in History and Philosophy of Science Part B: Studies in History and Philosophy of Modern Physics, 35, 1-30. http://dx.doi.org/10.1016/j.shpsb.2001.11.001

[20] Laughlin, R.B. (2005) A Different Universe: Reinventing Physics from the Bottom Down. Basic, New York, 54.

[21] Goldstein, S. (2013) Bohmian Mechanics. The Stanford Encyclopedia of Philosophy. http://plato.stanford.edu/archives/spr2013/entries/qm-bohm/

[22] Abächerli, A. (2015) Au-delà des paradoxes de la connaissance (Meaning "Beyond the Paradoxes of Knowledge”). Comment l'hypothèse de vrais infinis dans le monde peut éliminer les incohérences des sciences de la connaissance. Éditions Universitaires Européennes, Saarbrücken, p. 90, p. 152.

[23] Abächerli, A. (2014) On Perception and Some Consequences: The World, the Brain and Infinity. International Journal of Philosophy, 2, 60-71.

[24] Abächerli, A. (2015) Au-delà des paradoxes de la connaissance (Meaning "Beyond the Paradoxes of Knowledge”). Comment l’hypothèse de vrais infinis dans le monde peut éliminer les incohérences des sciences de la connaissance. Éditions Universitaires Européennes, Saarbrücken.

[25] Abächerli, A. (2016) The Open Brain in the Open World: Beyond the Paradoxes of Knowledge.

[26] Laughlin, R.B. (2005) A Different Universe: Reinventing Physics from the Bottom Down. Basic, New York, 36. 
[27] Kim, J. (1992) Downward Causation in Emergentism and Non-Reductive Physicalism. In: Beckermann, A., Flohr, H. and Kim, J., Eds., Emergence or Reduction? Essays on the Prospects of Nonreductive Physicalism, Walter de Gruyter, Berlin, 119-138.

[28] Kim, J. (1993) The Non-Reductivist’s Troubles with Mental Causation. In: Heil, J. and Mele, A., Eds., Mental Causation, Oxford University Press, Oxford, 189-210.

[29] Galaaen, Ø.S. (2007) The Disturbing Matter of Downward Causation. PhD Dissertation, University of Oslo, Oslo.

[30] Laughlin, R.B. (2005) A Different Universe: Reinventing Physics from the Bottom Down. Basic, New York, 19.

[31] Laughlin, R.B. (2005) A Different Universe: Reinventing Physics from the Bottom Down. Basic, New York, 171.

[32] Healey, R. (2009) Holism and Nonseparability in Physics. The Stanford Encyclopedia of Philosophy, Chapter 3. http://plato.stanford.edu/archives/spr2009/entries/physics-holism/

[33] Cushing, J.T. (1982) Models and Methodologies in Current Theoretical High-Energy Physics. Synthese, 50, 5-101. http://dx.doi.org/10.1007/bf00413723

[34] Quine, W.V.O. (1964) From a Logical Point of View. Harvard University Press, Cambridge.

[35] Carnap, R. (1934) Der Logische Syntax der Sprache. Springer, Wien. http://dx.doi.org/10.1007/978-3-662-25375-5

[36] Carnap, R. (1942) Introduction to Semantics. Harvard University Press, Cambridge.

[37] Laughlin, R.B., and Pines, D. (2000) The Theory of Everything. Proceedings of the National Academy of Sciences of the United States of America, 97, 30. http://dx.doi.org/10.1073/pnas.97.1.28

[38] Laughlin, R.B., and Pines, D. (2000) The Theory of Everything. Proceedings of the National Academy of Sciences of the United States of America, 97, 32. http://dx.doi.org/10.1073/pnas.97.1.28

[39] Laughlin, R.B. (2005) A Different Universe: Reinventing Physics from the Bottom Down. Basic, New York, 45.

[40] Laughlin, R.B. (2005) A Different Universe: Reinventing Physics from the Bottom Down. Basic, New York, 44.

[41] Laughlin, R.B. (2005) A Different Universe: Reinventing Physics from the Bottom Down. Basic, New York, 208-209.

[42] Laughlin, R.B. (2005) A Different Universe: Reinventing Physics from the Bottom Down. Basic, New York, 9.

[43] Anderson, P.W. (1972) More Is Different. Science, 177, 393. http://dx.doi.org/10.1126/science.177.4047.393

[44] Bishop, R.C. (2008) Downward Causation in Fluid Convection. Synthese, 160, 229-248. http://dx.doi.org/10.1007/s11229-006-9112-2

[45] Anderson, P.W. (1972) More Is Different. Science, 177, 393-396. http://dx.doi.org/10.1126/science.177.4047.393

[46] Laughlin, R.B. (2005) A Different Universe: Reinventing Physics from the Bottom Down. Basic, New York, 46.

[47] Laughlin, R.B. (2005) A Different Universe: Reinventing Physics from the Bottom Down. Basic, New York, 41.

[48] Laughlin, R.B. (2005) A Different Universe: Reinventing Physics from the Bottom Down. Basic, New York, 43.

[49] Laughlin, R.B. (2005) A Different Universe: Reinventing Physics from the Bottom Down. Basic, New York, 49.

[50] Laughlin, R.B. and Pines, D. (2000) The Theory of Everything. Proceedings of the National Academy of Sciences, 97, 28. http://dx.doi.org/10.1073/pnas.97.1.28

[51] Healey, R. (2009) Holism and Nonseparability in Physics. The Stanford Encyclopedia of Philosophy, Chapter 2. http://plato.stanford.edu/archives/spr2009/entries/physics-holism/

[52] Laughlin, R.B. (2005) A Different Universe: Reinventing Physics from the Bottom Down. Basic, New York, 7.

[53] Bryson, B. (2004) A Short History of Nearly Everything. Transworld, London, 453.

[54] Davies, P. (1999) The Fifth Miracle: The Search for the Origin of Life. Penguin, London, 127.

[55] Greene, B. (2003) The Elegant Universe. W.W. Norton, New York, 142.

[56] Campbell, D.T. (1974) Downward Causation in Hierarchically Organised Biological Systems. In: Ayala, F.J. and Dobzhansky, T., Eds., Studies in the Philosophy of Biology: Reduction and Related Problems, Macmillan, London, 179-186. http://dx.doi.org/10.1007/978-1-349-01892-5_11

[57] Laughlin, R.B. (2005) A Different Universe: Reinventing Physics from the Bottom Down. Basic, New York, 201.

[58] Bryson, B. (2004) A Short History of nearly Everything. Transworld, London, 28.

[59] Bryson, B. (2004) A Short History of nearly Everything. Transworld, London, 60-61.

[60] Bennett, K. (2010) The Theory of Evolution. New Scientist, 208, 28-31. http://dx.doi.org/10.1016/S0262-4079(10)62530-4

[61] Abächerli, A. (2014) On Perception and Some Consequences: The World, the Brain and Infinity. International Journal of Philosophy, 2, 64-68. 
[62] Diacu, F. and Philip H. (1996) Celestial Encounters: The Origin of Chaos and Stability. Princeton University Press, Princeton.

[63] Dodelson, S. (2003) Modern Cosmology. Academic Press, San Diego.

[64] Wikipedia. Structure Formation. http://en.wikipedia.org/wiki/Structure_formation

[65] Padmanabhan, T. (1993) Structure Formation in the Universe. Cambridge University Press, Cambridge, 415. 